Meta

Journal des traducteurs

Translators' Journal

\title{
De Pénélope à la midinette
}

\section{Robert Dubuc}

Volume 19, numéro 3, septembre 1974

URI : https://id.erudit.org/iderudit/002633ar

DOI : https://doi.org/10.7202/002633ar

Aller au sommaire du numéro

Éditeur(s)

Les Presses de l'Université de Montréal

ISSN

0026-0452 (imprimé)

1492-1421 (numérique)

Découvrir la revue

Citer cet article

Dubuc, R. (1974). De Pénélope à la midinette. Meta, 19(3), 147-150.

https://doi.org/10.7202/002633ar

Ce document est protégé par la loi sur le droit d'auteur. L'utilisation des services d'Érudit (y compris la reproduction) est assujettie à sa politique d'utilisation que vous pouvez consulter en ligne.

https://apropos.erudit.org/fr/usagers/politique-dutilisation/
Cet article est diffusé et préservé par Érudit.

Érudit est un consortium interuniversitaire sans but lucratif composé de l’Université de Montréal, l'Université Laval et l'Université du Québec à Montréal. Il a pour mission la promotion et la valorisation de la recherche. https://www.erudit.org/fr/ 


\section{PROBLÈMES ET SOLUTIONS}

\section{DE PÉNÉLOPE À LA MIDINETTE}

Le tissage et le métier à tisser sont des institutions vieilles comme le monde, et si l'un et l'autre ont connu de multiples perfectionnements, les opérations fondamentales qu'ils impliquent n'ont pas évolué sensiblement.

Le tissage se ramène à trois opérations de base que Linton nomme shedding, picking et beating-up. Le shedding, mouvement des lames, consiste à ouvrir en angle les deux nappes élémentaires de fils de chaîne de façon à permettre l'insertion du fil de trame. C'est justement cette insertion du fil de trame au moyen de la navette qui constitue la chasse (picking). Une fois cette opération accomplie, il faut ramener la duite, ou fil de trame, contre le tissu précédemment constitué : c'est la frappe (beating-up).

Le mot picking, appliqué au domaine du textile, mérite qu'on s'y arrête, car il constitue un exemple assez rare de polysémie au sein d'une même aire de vocabulaire. À l'étape de la récolte du coton, le mot désigne l'action de détacher les capsules du cotonnier. C'est ce qu'on appelle en français la cueillette. Lorsque le coton, après avoir été mis en balle, arrive à l'usine, il subit, après les opérations d'ouverture, un traitement, appelé également picking, qui vise à le débarrasser des impuretés et des nœuds afin de permettre la préparation des nappes en vue du filage. Dans ce sens, ce mot correspond à floconnage. On voit les risques de collision sémantique que cette polysémie peut présenter.

Pour revenir au tissage, il se fait, chacun le sait, grâce à un appareil nommé métier (loom). Le métier fondamentalement se compose de divers éléments que nous allons tenter d'identifier.

Il comprend d'abord l'ensouple (warp beam) sur laquelle on charge les fils de chaîne (warp).En quittant l'ensouple, la chaîne passe sur un porte-fil ( whip roll) qui la ramène à l'horizontale. 
Pour faciliter le repérage des brins en cas de brisure, les fils de chaîne passent entre des baguettes dites d'envergure ou d'encroix (lease rods), puis ils sont ensuite enfilés dans les maillons (eye, eyelet) des lisses (heddles), maintenues dans un cadre appelé harnais ou lame (harness frame). Ces lames ou harnais s'élèvent ou s'abaissent pour former la foule (shed) de façon à permettre le passage du fil de trame (filling, weft, woof). Des pédales ou marches actionnent les lames au moyen d'excentriques (cam) et de cuirs (harness strap). Ces derniers s'engagent dans le galet de contre-effet (harness roller), situé à la partie supérieure du métier. Le fil de chaîne, enroulé sur une bobine (bobbin), est porté par la navette (shuttle) mise en action par le battant (lay). Le battant se compose, à son tour, de deux épées de chasse (lay sword) qui font passer la navette dans les boîtes à navette (shuttle box) situées de part et d'autre du métier. La navette court sur le sommier du battant (raceplate) auquel s'articule le peigne (reed) qui vient frapper ou battre la duite (pick) contre le tissu déjà constitué. Le peigne est surmonté d'un chapeau de battant (reed cap) qui en permet le mouvement. $\mathrm{Au}$ fur et à mesure que le tissu se construit, il vient passer contre la poitrinière (breast beam) par l'action du rouleau d'appel (sand roll) avant d'aller s'enrouler sur le rouleau à tissu (cloth roll), nommé communément rouleau tout simplement.

Notre petite excursion dans le domaine du tissage nous a permis d'amasser les éléments d'un petit vocabulaire dont il nous reste à dégager les notions de façon plus précise. (Les chiffres qui suivent chaque terme renvoient à la bibliographie.)

beating-up 4

frappe 1

Opération du tissage qui consiste à battre la duite contre le tissu déjà constitué, au moyen d'un peigne prévu à cette fin.

breast beam 5

poitrinière 1

Partie du métier à tisser sur laquelle passe le tissu, tiré par le rouleau d'appel, avant d'aller s'enrouler sur le rouleau à tissu.

cam 5

excentrique (n.f.) 3

Plaque de forme irrégulière actionnée par les pédales du métier et qui commande le mouvement des lames.

chain 6 v. warp

cloth beam $4 \quad$ rouleau à tissu (1-7)

Cylindre où s'enroule le tissu constitué.

eye; eyelet 4

maillon 1

Trou situé au centre de chaque lisse et dans lequel on passe un fil de trame.

harness; harness frame 6 harnais; lame (3-1)

Cadre supportant les lisses dans lesquelles passent les fils de trame.

hamess roller 5

galet de contre-effet 2

Petite poulie située à la partie supérieure du métier et dont l'action se conjugue à celle des excentriques pour assurer les mouvements successifs des lames.

harness strap 5

cuir 3

Courroie supportant les harnais ou lames à leur partie supérieure. 
heddle 4

lisse 1

Tige métallique comprenant en son centre un maillon dans lequel passe un fil de trame.

heddle eye v. eye

lay 5

battant 1

Mécanisme du métier qui commande à la fois le mouvement de la navette et la frappe de la duite.

lay sword 5

épée de chasse 2

Pièce métallique verticale supportant le battant du métier.

lease rod 5

baguette d'envergure 2

baguette d'encroix 3

Baguette de bois ou de verre qui divise la nappe de fils de chaîne en nappes élémentaires, pour permettre de retrouver facilement la place exacte des fils qui viendraient à casser.

pick 5

duite 3

Nom que prend le fil de trame laissé par le passage de la navette dans la foule.

picker stick 5

sabre 2

Dispositif placé de chaque côté du métier et qui assure le passage de la navette dans chaque foule.

1. picking 5

cueillette 7

À la récolte du coton, opération par laquelle les capsules sont détachées du cotonnier.

2. picking 5

floconnage 7

Après l'ouverture des balles, opération de nettoyage et d'épuration qui rend au coton sa texture floconneuse pour former les nappes servant au filage.

3. picking 5

chasse 3

Passage de la navette d'une boîte à l'autre de façon à laisser dans chaque foule une duite que le peigne vient ensuite frapper contre le tissu déjà constitué.

raceplate 5

sommier du battant 1

semelle du battant $?$

Traverse qui forme la partie inférieure du battant et sur laquelle glisse la navette.

reed 4

peigne 1

Dispositif formé de dents de métal ou de roseau qui sert à séparer les fils de chaîne et à battre le fils de trame contre le tissu constitué.

reed cap 5

chapeau de battant 3

Tringle horizontale placée à la partie supérieure du peigne pour permettre les mouvements de frappe.

sand roll 5

rouleau d'appel 1

Cylindre de bois recouvert d'une substance antidérapante et qui entraîne le tissu constitué vers le rouleau à tissu.

set of yarns 4

nappe de fils 1

Ensemble de fils non tissés. 
shed 4

foule (n.f.) 1

Angle formé par les mouvements de lames du métier en sens contraire de façon à permettre l'insertion de la duite.

shedding 5

mouvements des lames 7

Abaissement et relèvement successifs des lames de façon à former une foule.

sheet of warp 6

nappe de fils de chaîne 1

Ensemble de fils de chaîne solidaires d'une lame.

shuttle 4

navette 1

Enveloppe, faite en général de bois, contenant la bobine de fil de trame et qui laisse derrière elle une duite à chaque passage dans la foule.

shuttle box $5 \quad$ boîte à navette 1

Réceptacle, situé de part et d'autre du métier, où la navette va se loger après avoir traversé la foule.

treadle 5

pédale 1 ; marche 2

Levier commandant le mouvement des lames au moyen d'excentriques.

warp 5

fil de chaîne 1

Fil placé dans le sens de la longueur du métier. Les fils de chaîne sont placés parallèlement et sont régulièrement espacés.

warp beam 4 ensouple (n.f.) 1

Cylindre sur lequel on enroule la nappe de fils de chaîne après l'encollage.

weaving shed 4 v. shed

whip roll 5

porte-fil 2

Cylindre de faible section dominant l'ensouple et qui donne à la chaîne une trajectoire horizontale.

ROBERT DUBUC

\section{BIBLIOGRAPHIE}

1. Grand Larousse encyclopédique, Paris, Larousse, 1960.

2. Larousse trois volumes en couleur, Paris, Larousse, «In quarto Larousse », 1969.

3. Thiébaut, Raymond, le Tissage, Paris, Presses universitaires de France, «Que sais-je ?», $\mathrm{n}^{\circ} 546,1964,126 \mathrm{p}$.

4. Stout, Evelyn E., Introduction to Textiles, $3^{e}$ éd., New York, John Wiley \& Sons, 1970, $434 \mathrm{p}$.

5. Linton, George E., The Modern Textile Dictionary, $2^{\mathrm{e}}$ éd., New York, Dueli, Sloan and Pearce, 1963, $1077 \mathrm{p}$.

6. Encyclopaedia britannica, Chicago, William Benton Publisher, 1970.

7. Fiches de la Banque de terminologie de l'Université de Montréal. 